\title{
On the Use of A Priori Knowledge in Pattern Search Methods: Application to Beam Angle Optimization for Intensity-Modulated Radiation Therapy
}

\author{
Humberto Rocha ${ }^{1}$, Joana M. Dias ${ }^{1,2}$, Brigida C. Ferreira ${ }^{3,4}$, and \\ Maria do Carmo Lopes ${ }^{3,4}$ \\ 1 INESC-Coimbra, Rua Antero de Quental, 199 \\ 3000-033 Coimbra, Portugal \\ 2 Faculdade de Economia, Universidade de Coimbra, \\ 3004-512 Coimbra, Portugal \\ 3 I3N, Departamento de Física, Universidade de Aveiro, \\ 3810-193 Aveiro, Portugal \\ 4 Serviço de Física Médica, IPOC-FG, EPE, \\ 3000-075 Coimbra, Portugal \\ hrocha@mat.uc.pt, joana@fe.uc.pt, brigida@ua.pt, \\ mclopes@ipocoimbra.min-saude.pt
}

\begin{abstract}
Pattern search methods are widely used for the minimization of non-convex functions without the use of derivatives. One of the main features of pattern search methods is the flexibility to incorporate different search strategies taking advantage of the imported global optimization techniques without jeopardizing their convergence properties. Pattern search methods can also be adapted to problem contexts where the user can provide points incorporating a priori knowledge of the problem that can lead to an objective function improvement. Here, an automated incorporation of a priori knowledge in pattern search methods is implemented instead of an algorithm that requires the user's contribution. Moreover, a priori knowledge can also play a role on the choice of the initial point(s), an important aspect in the success of a global optimization process. Our pattern search approach is tailored for addressing the beam angle optimization (BAO) problem in intensity-modulated radiation therapy (IMRT) treatment planning that consists of selecting appropriate radiation incidence directions and may influence the quality of the IMRT plans, both to enhance better organs sparing and to improve tumor coverage. Beam's-eye-view dose ray tracing metrics are used as a priori knowledge of the problem both to decide the initial point(s) and to be incorporated within a pattern search methods framework. A couple of retrospective treated cases of head-and-neck tumors at the Portuguese Institute of Oncology of Coimbra is used to discuss the benefits of incorporating a priori dosimetric knowledge in pattern search methods for the optimization of the BAO problem.
\end{abstract}

Keywords: Pattern Search Methods, Beam's-Eye-View Dose Metrics, IMRT, Beam Angle Optimization 


\section{Introduction}

Pattern search methods are widely used for the minimization of non-convex functions without the use of derivatives or approximations to derivatives. Pattern search methods are organized around two steps at every iteration: the poll step and the search step. The poll step guarantees global convergence to stationary points by performing a local search in a neighborhood around the current iterate using the concepts of positive bases. The search step consists of a search away from the current iterate, free of rules as long as the search is finite. One of the main features of pattern search methods is the flexibility to incorporate different search strategies in the search step, taking advantage of the imported global optimization techniques, without jeopardizing their convergence properties. Different techniques for global optimization have been successfully incorporated on the search step, including surrogate optimization, e.g., radial basis functions [15], or global optimization, e.g., particle swarm optimization [19]. Pattern search methods can also be adapted to problem contexts where the user can provide points incorporating physical or a priori knowledge of the problem that can lead to an objective function decrease [1]. Here, an automated incorporation of a priori knowledge in pattern search methods is implemented instead of an algorithm that requires the user's contribution. Moreover, a priori knowledge can also play a role on the choice of the initial point(s), an important aspect in the success of a global optimization process.

The pattern search approach presented in this work is tailored for addressing the beam angle optimization (BAO) problem in intensity-modulated radiation therapy treatment planning. The intensity-modulated radiation therapy (IMRT) is a modern type of radiation therapy, whose planning leads to complex optimization problems, including the BAO problem - the problem of deciding which incidence radiation beam angles should be used. The pattern search methods framework has been used to address the BAO problem successfully due to its ability to avoid local entrapment and its need for few function value evaluations to converge $[14,15]$. Here, a priori knowledge of the problem is incorporated in pattern search methods using beam's-eye-view dose ray tracing metrics. The beam's-eye-view concept considers topographic and/or dosimetric criteria to rank the candidate beam directions.

The goal of the paper is twofold: to discuss the influence of a priori dose metric knowledge on the choice of the initial point(s) and to discuss the benefits of incorporating a priori knowledge in pattern search methods. A couple of retrospective treated cases of head-and-neck tumors at the Portuguese Institute of Oncology of Coimbra is used to discuss these benefits for the optimization of the BAO problem. Our approach is tailored to address this particular problem but it can be easily extended for other general problems. The paper is organized as follows. In the next Section we describe the BAO problem. Beam's-eye-view dose ray tracing metrics and its use within the pattern search methods framework is presented in Section 3. Computational tests using clinical examples of head-and-neck cases are presented in Section 4. In the last Section we have the conclusions. 


\section{Beam Angle Optimization in IMRT Treatment Planning}

The BAO problem consists on the selection of appropriate radiation incidence directions in radiation therapy treatment planning and may be decisive for the quality of the treatment plan, both for appropriate tumor coverage and for enhance better organs sparing. Many attempts to address the BAO problem can be found in the literature including simulated annealing [3], genetic algorithms [9], particle swarm optimization [11] or other heuristics incorporating a priori knowledge of the problem [13]. The BAO problem is quite difficult, and yet to be solved in a satisfactory way, since it is a highly non-convex optimization problem with many local minima [4].

In IMRT, the radiation beam is modulated by a multileaf collimator, transforming the beam into a grid of smaller beamlets of independent intensities, allowing the irradiation of the patient using non-uniform radiation fields from selected angles aiming to deliver a dose of radiation to the tumor minimizing the damages on the surrounding healthy organs and tissues. The IMRT treatment planning is usually a sequential process where initially a given number of beam directions are selected followed by the fluence map optimization (FMO) at those beam directions. Obtaining the optimal fluences for a given beam angle set is time consuming due to the dosimetric calculations required. For that reason, many of the previous BAO studies are based on a variety of scoring methods or approximations to the FMO to gauge the quality of the beam angle set. However, when the BAO problem is not based on the optimal FMO solutions, the resulting beam angle set has no guarantee of optimality and has questionable reliability. Therefore, our approach for modeling the BAO problem uses the optimal solution value of the FMO problem as the measure of the quality for a given beam angle set. Thus, we will present the formulation of the BAO problem followed by the formulation of the FMO problem we used. Here, we will assume that the number of beam angles is defined a priori by the treatment planner and that all the radiation directions lie on the same plane.

\subsection{BAO Model}

Let us consider $n$ to be the fixed number of (coplanar) beam directions, i.e., $n$ beam angles are chosen on a circle around the CT-slice of the body that contains the isocenter (usually the center of mass of the tumor). In our formulation, instead of a discretized sample, all continuous $\left[0^{\circ}, 360^{\circ}\right]$ gantry angles will be considered. Since the angle $-10^{\circ}$ is equivalent to the angle $350^{\circ}$ and the angle $370^{\circ}$ is the same as the angle $10^{\circ}$, we can avoid a bounded formulation. A simple formulation for the BAO problem is obtained by selecting an objective function such that the best set of beam angles is obtained for the function's minimum:

$$
\begin{aligned}
& \min f\left(\theta_{1}, \ldots, \theta_{n}\right) \\
& \text { s.t. }\left(\theta_{1}, \ldots, \theta_{n}\right) \in \mathbb{R}^{n} .
\end{aligned}
$$


Here, for the reasons stated before, the objective $f\left(\theta_{1}, \ldots, \theta_{n}\right)$ that measures the quality of the set of beam directions $\theta_{1}, \ldots, \theta_{n}$ is the optimal value of the FMO problem for each fixed set of beam directions. The FMO model used is presented next.

\section{$2.2 \quad$ FMO Model}

In order to solve the FMO problem, i.e., to determine optimal fluence maps, the radiation dose distribution deposited in the patient needs to be assessed accurately. Each structure's volume is discretized into small volume elements (voxels) and the dose is computed for each voxel considering the contribution of each beamlet. Typically, a dose matrix D is constructed from the collection of all beamlet weights, by indexing the rows of $\mathrm{D}$ to each voxel and the columns to each beamlet, i.e., the number of rows of matrix $\mathrm{D}$ equals the number of voxels $\left(N_{v}\right)$ and the number of columns equals the number of beamlets $\left(N_{b}\right)$ from all beam directions considered. Therefore, using matrix format, we can say that the total dose received by the voxel $i$ is given by $\sum_{j=1}^{N_{b}} D_{i j} w_{j}$, with $w_{j}$ the weight of beamlet $j$. Usually, the total number of voxels is large, reaching the tens of thousands, which originates large-scale problems. This is one of the main reasons for the difficulty of solving the FMO problem.

For a given beam angle set, an optimal IMRT plan is obtained by solving the FMO problem - the problem of determining the optimal beamlet weights for the fixed beam angles. Many mathematical optimization models and algorithms have been proposed for the FMO problem, including linear models [18], mixed integer linear models [10] and nonlinear models [2]. Here, we will use this later approach that penalizes each voxel according to the square difference of the amount of dose received by the voxel and the amount of dose desired/allowed for the voxel. This formulation yields a quadratic programming problem with only linear non-negativity constraints on the fluence values [18]:

$$
\begin{aligned}
& \min _{w} \sum_{i=1}^{N_{v}} \frac{1}{v_{S}}\left[\underline{\lambda}_{i}\left(T_{i}-\sum_{j=1}^{N_{b}} D_{i j} w_{j}\right)_{+}^{2}+\bar{\lambda}_{i}\left(\sum_{j=1}^{N_{b}} D_{i j} w_{j}-T_{i}\right)_{+}^{2}\right] \\
& \text { s.t. } \quad w_{j} \geq 0, j=1, \ldots, N_{b},
\end{aligned}
$$

where $T_{i}$ is the desired dose for voxel $i$ of the structure $v_{S}, \underline{\lambda}_{i}$ and $\bar{\lambda}_{i}$ are the penalty weights of underdose and overdose of voxel $i$, and $(\cdot)_{+}=\max \{0, \cdot\}$. This nonlinear formulation implies that a very small amount of underdose or overdose may be accepted in clinical decision making, but larger deviations from the desired/allowed doses are decreasingly tolerated [2].

The FMO model is used as a black-box function and the conclusions drawn regarding $\mathrm{BAO}$ coupled with this nonlinear model are valid also if different FMO formulations are considered. 


\section{Pattern Search Methods Incorporating A Priori Knowledge}

The incorporation of a priori knowledge in pattern search methods is done using beam's-eye-view dose ray tracing metrics. We will briefly describe the concept of beam's-eye-view and the strategy used to take advantage of the incorporation of the resulting metrics in the pattern search method framework applied to the BAO problem.

\subsection{Beam's-eye-view Dose Metrics}

Conventional beam's-eye-view (BEV) tools consider only geometric criteria, i.e., topographic localization of tumor volume(s) versus surrounding healthy structures, to evaluate each candidate beam direction. The use of beam's-eyeview dose metrics (BEVD) was introduced by Pugachev and Xing [12] to evaluate and rank the irradiation beam directions using a score function that accounts for beam modulation unlike the traditional BEV. An intensity-modulated beam can intercept a large volume of an organ at risk (OAR) or normal tissue and may not be necessarily a bad beam direction, which makes the geometrical criteria used by BEV limited. The computation of a metric to gauge the quality of incidence radiation directions should also consider the dose tolerances of the involved structures. Thus, in IMRT, a score function based on dosimetric criteria is more appropriate to measure the quality of a radiation beam direction.

The measure of the quality of a radiation beam direction adopted is based on sensitive structures tolerance dose as a determinant factor for deliverable target dose. A given incidence radiation direction is preferred if it can deliver more dose to the target without exceeding the tolerance dose of the OARs or normal tissue located on the path of the beam $[12,13]$. The BEVD score for a given beam angle corresponds to the computation of the maximum achievable intensity for each beamlet involved, which depends on the locations and tolerances of the OARs along the path of the beamlet.

The BEVD score calculation of a beam requires the assumption of a single incident beam. Initially, all beamlets are assigned with an intensity that assures the delivery of a dose that fulfills the prescription to every target voxel. Beamlet intensities are then iteratively updated until tolerance dose for every structure's voxel crossed by the all the beamlets is not exceeded. The intensities obtained for each beamlet correspond to the maximum usable intensity of the beamlet without exceeding the tolerance of the sensitive structures. Finally, a forward dose calculation using the maximum usable beamlet intensities is performed and the score of a given beam direction is computed $[12,13]$ :

$$
S_{k}=\frac{1}{N_{T}} \sum_{i \in \text { Target }}\left(\frac{d_{i k}}{D_{P}^{T}}\right)^{2},
$$

where $N_{T}$ is the number of voxels in the target, $D_{P}^{T}$ is the target prescription dose and $d_{i k}$ is the "maximum" dose delivered to the target voxel $i$ by the radiation beam direction $k$. 
The BEVD score is based on an intuitive consideration of the deliverable dose capability to the target of a single beam direction. This information can be used to construct initial point(s) whose neighborhood may be worth of being thoroughly explored. However, the optimal beam configuration for an IMRT treatment should balance the BEVD score and the beam interplay as a result of the overlap of radiation fields. Thus, BEVD scores are used as a priori knowledge to construct an insightful algorithm for beam angle optimization. This a priori knowledge of the problem is used by a pattern search methods framework.

\subsection{Pattern Search Methods Incorporating BEVD}

Pattern search methods are derivative-free optimization methods that use the directions of positive bases to explore the search space, such that iterate progression is solely based on a finite number of function evaluations in each iteration, without explicit or implicit use of derivatives. We will briefly describe pattern search methods for unconstrained optimization problems such as the beam angle problem formulated in (1).

Pattern search methods use the concept of positive bases (or positive spanning sets) to move towards a direction that would produce a function decrease. A positive basis for $\mathbb{R}^{n}$ can be defined as a set of nonzero vectors of $\mathbb{R}^{n}$ whose positive combinations span $\mathbb{R}^{n}$ (positive spanning set), but no proper set does. The motivation for directional direct search methods such as pattern search methods is given by one of the main features of positive basis (or positive spanning sets) [7]: there is always a vector $\mathbf{v}^{i}$ in a positive basis (or positive spanning set) that is a descent direction unless the current iterate is at a stationary point, i.e., there is an $\alpha>0$ such that $f\left(x^{k}+\alpha \mathbf{v}^{i}\right)<f\left(x^{k}\right)$. This is the core of directional direct search methods and in particular of pattern search methods.

Pattern search methods are iterative methods generating a sequence of nonincreasing iterates $\left\{x_{k}\right\}$. Given the current iterate $x^{k}$, at each iteration $k$, the next point $x^{k+1}$, aiming to provide a decrease of the objective function, is chosen from a finite number of candidates on a given mesh $M_{k}$ defined as

$$
M_{k}=\left\{x^{k}+\alpha_{k} \mathbf{V} \mathbf{z}: \mathbf{z} \in \mathbb{Z}_{+}^{p}\right\},
$$

where $\alpha_{k}$ is the mesh-size (or step-size) parameter, $\mathbb{Z}_{+}$is the set of nonnegative integers and $\mathbf{V}$ denote the $n \times p$ matrix whose columns correspond to the $p$ $(\geq n+1)$ vectors forming a positive spanning set.

Pattern search methods consider two steps at every iteration. The first step consists of a finite search on the mesh, free of rules, with the goal of finding a new iterate that decreases the value of the objective function at the current iterate. This step, called the search step, has the flexibility to use any strategy, method or heuristic, or take advantage of a priori knowledge of the problem at hand, as long as it searches only a finite number of points in the mesh. The search step provides the flexibility for a global search since it allows searches away from the neighborhood of the current iterate, and influences the quality of the local minimizer or stationary point found by the method. If the search step fails to 
produce a decrease in the objective function, a second step, called the poll step, is performed around the current iterate. The poll step follows stricter rules and, using the concepts of positive bases, attempts to perform a local search in a mesh neighborhood around $x^{k}, \mathcal{N}\left(x^{k}\right)=\left\{x^{k}+\alpha_{k} \mathbf{v}\right.$ : for all $\left.\mathbf{v} \in P_{k}\right\} \subset M_{k}$, where $P_{k}$ is a positive basis chosen from the finite positive spanning set $\mathbf{V}$. For a sufficiently small mesh-size parameter $\alpha_{k}$, the poll step is guaranteed to provide a function reduction, unless the current iterate is at a stationary point [1]. So, if the poll step also fails to produce a function reduction, the mesh-size parameter $\alpha_{k}$ must be decreased. On the other hand, if the search or the poll steps obtain an improved value for the objective function, the mesh-size parameter is increased or held constant.

The efficiency of pattern search methods improved significantly by reordering the poll directions according to descent indicators built from simplex gradients [6]. Here, the poll directions are reordered according to the BEVD scores meaning that directions with higher dosimetric value are tested first. Adding to the efficiency provided by an insightful reordering of the poll directions, the search step was recently provided with the use of minimum Frobenius norm quadratic models to be minimized within a trust region, which can lead to a significant improvement of direct search for smooth, piecewise smooth, and noisy problems [5]. The prior knowledge of the problem is also included in this step to take advantage of BEVD scores. A trial point is tested by considering the current best beam angle configuration and replacing the beam direction with smallest BEVD score by a beam direction with larger score that is not in the close neighborhood of the remaining beam directions. Last, but not least, the prior knowledge of the problem is used on the choice of initial point(s) by considering initial beam angle sets whose beam directions correspond to the largest BEVD scores. The strategy sketched is tailored for addressing the BAO problem taking advantage of prior knowledge of the problem:

\section{Algorithm 1 (PSM framework incorporating BEVD).}

0. Initialization Set $k=0$. Compute BEVD scores for each beam angle. Choose a positive spanning set $\mathbf{V}, \alpha_{0}>0$, and $x^{0} \in \mathbb{R}^{n}$ considering the beam directions with largest BEVD scores.

1. Search step Evaluate $f$ at a finite number of points in $M_{k}$ with the goal of decreasing the objective function value at $x^{k}$. If $x^{k+1} \in M_{k}$ is found satisfying $f\left(x^{k+1}\right)<f\left(x^{k}\right)$, go to step 4 . Both search step and iteration are declared successful. Otherwise, go to step 2 and search step is declared unsuccessful.

2. Poll step This step is only performed if the search step is unsuccessful. Reorder the poll directions according to the BEVD scores. If $f\left(x^{k}\right) \leq f(x)$ for every $x$ in the mesh neighborhood $\mathcal{N}\left(x^{k}\right)$, then go to step 3 and shrink $M_{k}$. Both poll step and iteration are declared unsuccessful. Otherwise, choose a point $x^{k+1} \in \mathcal{N}\left(x^{k}\right)$ such that $f\left(x^{k+1}\right)<f\left(x^{k}\right)$ and go to step 4. Both poll step and iteration are declared successful.

3. Mesh reduction Let $\alpha_{k+1}=\frac{1}{2} \times \alpha_{k}$. Set $k=k+1$ and return to step 1 .

4. Mesh expansion Let $\alpha_{k+1}=\alpha_{k}$. Set $k=k+1$ and return to step 1 . 


\section{Computational Results for Head-and-neck Clinical Examples}

Our tests were performed on a 2.66Ghz Intel Core Duo PC with 3 GB RAM. In order to facilitate convenient access, visualization and analysis of patient treatment planning data, as well as dosimetric data input for treatment plan optimization research, the computational tools developed within MATLAB and CERR - computational environment for radiotherapy research [8] are used widely for IMRT treatment planning research.

The incorporation of BEVD into the pattern search methods framework was tested using two clinical examples of retrospective treated cases of head-andneck tumors at the Portuguese Institute of Oncology of Coimbra (IPOC). In general, the head-and-neck region is a complex area to treat with radiotherapy due to the large number of sensitive organs in this region (e.g., eyes, mandible, larynx, oral cavity, etc.). For simplicity, in this study, the OARs used for treatment optimization were limited to the spinal cord, the brainstem and the parotid glands. The spinal cord and the brainstem are some of the most critical organs at risk (OARs) in the head-and-neck tumor cases. These are serial organs, i.e., organs such that if only one subunit is damaged, the whole organ functionality is compromised. Therefore, if the tolerance dose is exceeded, it may result in functional damage to the whole organ. Thus, it is extremely important not to exceed the tolerance dose prescribed for these type of organs. Other than the spinal cord and the brainstem, the parotid glands are also important OARs. The parotid gland is the largest of the three salivary glands. A common complication due to parotid glands irradiation is xerostomia (the medical term for dry mouth due to lack of saliva). This decreases the quality of life of patients undergoing radiation therapy of head-and-neck, causing difficulties to swallow. The parotids are parallel organs, i.e., if a small volume of the organ is damaged, the rest of the organ functionality may not be affected. Their tolerance dose depends strongly on the fraction of the volume irradiated. Hence, if only a small fraction of the organ is irradiated the tolerance dose is much higher than if a larger fraction is irradiated. Thus, for these parallel structures, the organ mean dose is generally used instead of the maximum dose as an objective for inverse planning optimization. The tumor to be treated plus some safety margins is called planning target volume (PTV). For the head-and-neck cases in study it was separated in two parts with different prescribed doses: PTV1 and PTV2. The prescription dose for the target volumes and tolerance doses for the OARs considered in the optimization are presented in Table 1.

The patients' CT sets and delineated structures are exported via Dicom RT to a freeware computational environment for radiotherapy research - CERR. We used CERR 3.2.2 version and MATLAB 7.4.0 (R2007a). An automatized procedure for dose computation for each given beam angle set was developed, instead of the traditional dose computation available from IMRTP module accessible from CERR's menubar. This automatization of the dose computation was essential for integration in our BAO algorithm. To address the convex nonlin- 
Table 1. Prescribed doses for all the structures considered for IMRT optimization.

\begin{tabular}{lccc}
\hline Structure & Mean dose & Max dose Prescribed dose \\
\hline Spinal cord & - & $45 \mathrm{~Gy}$ & - \\
Brainstem & - & $54 \mathrm{~Gy}$ & - \\
Left parotid & $26 \mathrm{~Gy}$ & - & - \\
Right parotid & $26 \mathrm{~Gy}$ & - & - \\
PTV1 & - & - & $70.0 \mathrm{~Gy}$ \\
PTV2 & - & - & $59.4 \mathrm{~Gy}$ \\
Body & - & $80 \mathrm{~Gy}$ & - \\
\hline
\end{tabular}

ear formulation of the FMO problem we used a trust-region-reflective algorithm (fmincon) of MATLAB 7.4.0 (R2007a) Optimization Toolbox.

We choose to implement the incorporation of BEVD scores into the pattern search methods framework taking advantage of the availability of an existing pattern search methods framework implementation used successfully by us to tackle the BAO problem $[15,16,17]$ - the last version of SID-PSM $[5,6]$. The spanning set used was the positive spanning set ([e-e $I-I]$. Each of these directions corresponds to, respectively, the rotation of all incidence directions clockwise, the rotation of all incidence directions counter-clockwise, the rotation of each individual incidence direction clockwise, and the rotation of each individual incidence direction counter-clockwise.

Treatment plans with five to nine equispaced coplanar beams are used at IPOC and are commonly used in practice to treat head-and-neck cases [2]. Therefore, treatment plans of five coplanar orientations were obtained using SID-PSM and using an algorithm that incorporates a priori knowledge in pattern search, denoted BEVD-PSM. These plans were compared with the typical 5-beam equispaced coplanar treatment plans denoted equi. Since we want to improve the quality of the typical equispaced treatment plans, a starting point considered is the equispaced coplanar beam angle set. The choice of this initial point and the non-increasing property of the sequence of iterates generated by pattern search methods imply that each successful iteration correspond to an effective improvement with respect to the usual equispaced beam configuration. A different initial point is considered using the BEVD scores. Beforehand, we need to compute the BEVD scores that will be the prior knowledge of the problem to be incorporated in the BAO optimization algorithm. For each patient, the scores for every beam angle are computed as described in Section 3.1. The initial point using the BEVD scores is obtained considering the peaks of the BEVD score curve that are not too close and correspond the solution obtained directly using BEVD criteria [12]. The obtained scores for the two patients and the corresponding initial points considered are displayed in Fig. 1.

The results of BAO optimization concerning the improvement of the objective function value for the two clinical cases of head-and-neck tumors using SID-PSM and BEVD-PSM considering the equispaced configuration (equi) and 


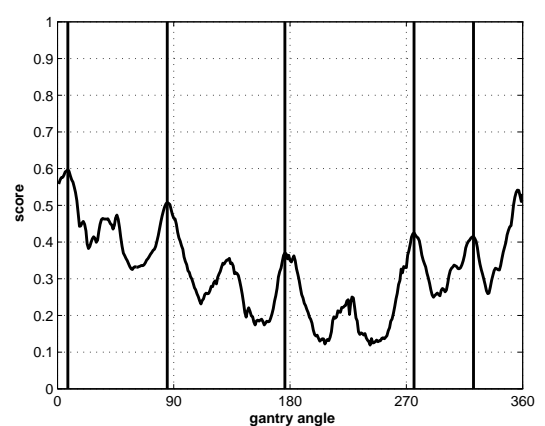

(a)

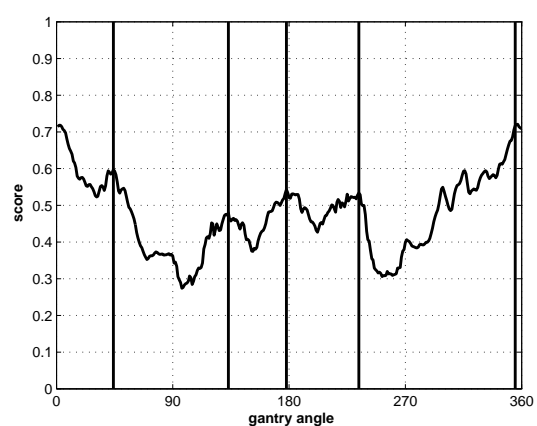

(b)

Fig. 1. BEVD scores as a function of the gantry angle and the BEVD initial point for cases 1 and 2,1(a) and 1 (b) respectively.

the beam's-eye-view configuration (bevd) as starting points are presented in Table 2. Overall, the comparison of the best beam angle configurations obtained by both approaches with the equispaced beam angle configuration in terms of objective function value is clearly favorable to the pattern search approaches, regardless of the initial point used. It is important to emphasize that the use of a priori knowledge through BEVD scores has a positive influence both in the choice of the initial point and also incorporated in the pattern search methods algorithm. That conclusion can be also withdrawn from the simple inspection of Fig. 2 where the performances of SID-PSM and BEVD-PSM are compared with respect to the objective function value decrease versus the number of function evaluations. The benefits of using an initial point that takes into account the dosimetric characteristics of the case at hand are highlighted by the comparison between SID-PSM starting with the equispaced configuration (equi) and the the beam's-eye-view configuration (bevd), favorable to the later. Even starting from higher function values, when the bevd configuration does not correspond to better function values compared with the equi configuration, as in case 2 , it seems to be advantageous to start from search regions where the neighbor beam directions of the initial configuration also have high BEVD scores. The advantage of incorporating BEVD scores in pattern search methods is clear since the best results are obtained by BEVD-PSM.

Despite the improvement in FMO value, the quality of the results can be perceived considering a variety of metrics. Typically, results are judged by their cumulative dose-volume histogram (DVH). The DVH displays the fraction of a structure's volume that receives at least a given dose. Another metric usually used for plan evaluation is the volume of PTV that receives $95 \%$ of the prescribed dose. Typically, $95 \%$ of the PTV volume is required. Using only 5 beam directions makes harder to obtain a satisfactory target coverage. DVH results for the two cases are displayed in Fig. 3. For clarity, the DVHs are split in PTV1 and PTV2 
Table 2. FMO value improvement obtained by SID-PSM and BEVD-PSM compared with the typical equispaced coplanar treatment plans, equi, considering the equispaced configuration (equi) and the beam's-eye-view configuration (bevd) as starting points.

\begin{tabular}{|c|c|c|c|c|c|c|c|}
\hline & equi & equi + & $I D-P S M$ & bevd + & SID- & bevd + & $B E V D-P S M$ \\
\hline ase & Fvalu & Fvalue & decrea & Fvalue & $\%$ de & Fvalue & $\%$ decrease \\
\hline 1 & 165.8 & 144.1 & $13.1 \%$ & 139.9 & 15 & 136.5 & \\
\hline 2 & 228.9 & 180.6 & $21.1 \%$ & 179.2 & $21.7 \%$ & 177.4 & $22.6 \%$ \\
\hline
\end{tabular}

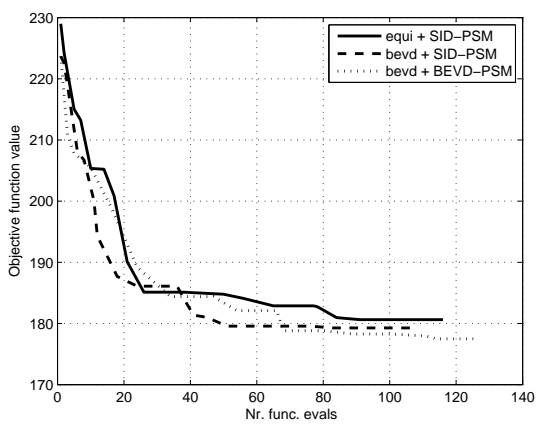

(a)

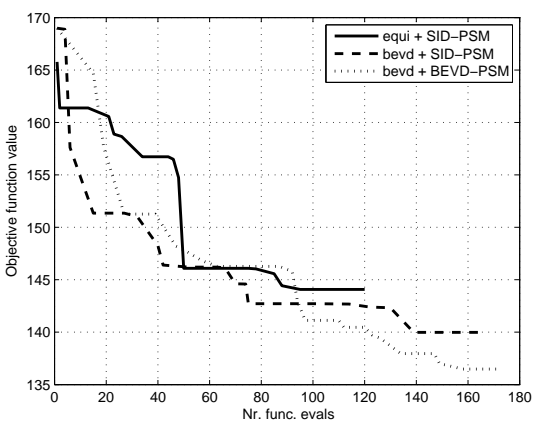

(b)

Fig. 2. History of the 5-beam angle optimization process using $S I D-P S M$ and BEVD$P S M$, considering the equispaced configuration (equi) and the beam's-eye-view configuration (bevd) as starting points, for cases 1 and 2, 2(a) and 2(b) respectively.

and the remaining structures distributed as an attempt to better visualize the results. The asterisks indicate $95 \%$ of PTV volumes versus $95 \%$ of the prescribed doses. The results displayed in Fig. 3 confirm the benefits of using the optimized beam directions obtained and used in BEVD-PSM treatment plans, with an improved target coverage and generally better organ sparing compared to the equispaced beam angle configuration, typically used in clinical practice.

\section{Conclusions}

The BAO problem is a continuous global highly non-convex optimization problem known to be extremely challenging and yet to be solved satisfactorily. This paper proposes an alternative approach to the $\mathrm{BAO}$ problem which is yet another step on the quest that may take us closer to find the global or near global optimum in a clinical acceptable time. The pattern search methods framework had already proved to be a suitable approach for the resolution of the non-convex $\mathrm{BAO}$ problem due to their structure, organized around two phases at every 

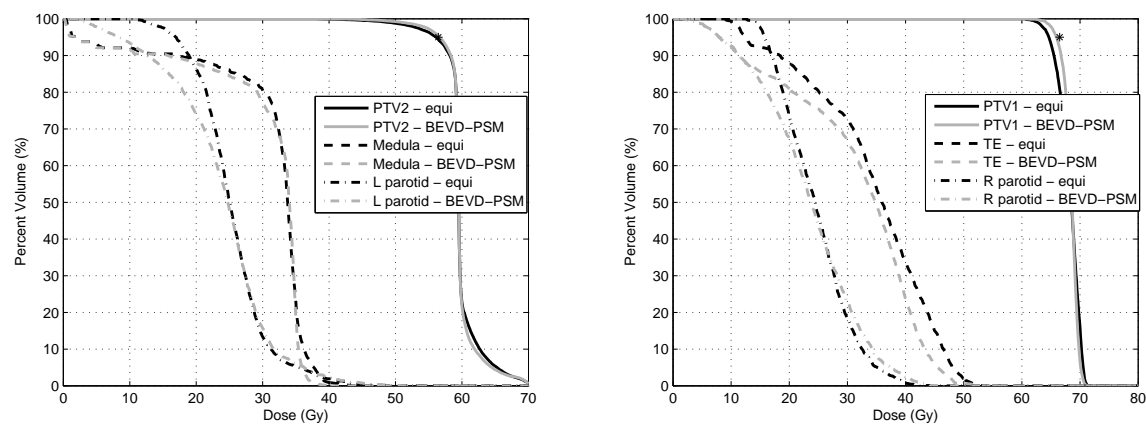

(a)
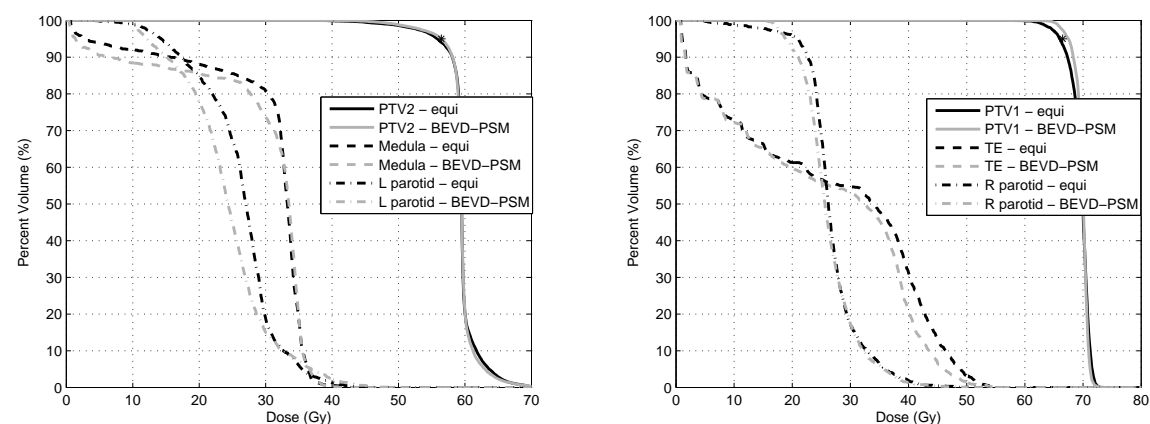

(b)

Fig. 3. Cumulative dose volume histogram comparing the results obtained by equi and $B E V D-P S M$ for cases 1 and 2, 3(a) and 3(b) respectively.

iteration. The poll step, where convergence to a local minima is assured, and the search step, where flexibility is conferred to the method since any strategy can be applied. The search step is provided with the use of minimum Frobenius norm quadratic models to be minimized within a trust region, which can lead to a significant improvement of direct search for the type of problems at hand. A novel approach for the resolution of the $\mathrm{BAO}$ problem, incorporating prior knowledge in a pattern search methods framework, was proposed and tested using a set of clinical head-and-neck cases. For the clinical cases retrospectively tested, the use of prior knowledge of the patient in our tailored approach showed a positive influence on the quality of the local minimizer found. The improvement of the solutions in terms of objective function value corresponded, for the head-andneck cases tested, to high quality treatment plans with better target coverage and with improved organ sparing. Moreover, the choice of the initial point also benefits from the prior knowledge of the problem leading to better solutions. 


\section{Acknowledgements}

This work was supported by QREN under Mais Centro (CENTRO-07-0224FEDER-002003) and FEDER funds through the COMPETE program and Portuguese funds through FCT under project grant PTDC/EIA-CCO/121450/2010. This work has also been partially supported by FCT under project grant PEstC/EEI/UI0308/2011. The work of H. Rocha was supported by the European social fund and Portuguese funds from MCTES.

\section{References}

1. Alberto, P., Nogueira, F., Rocha, H., Vicente, L.N.: Pattern search methods for user-provided points: Application to molecular geometry problems. SIAM J. Optim. 14, 1216-1236 (2004)

2. Aleman, D.M., Kumar, A., Ahuja, R.K., Romeijn, H.E., Dempsey, J.F.: Neighborhood search approaches to beam orientation optimization in intensity modulated radiation therapy treatment planning. J. Global Optim. 42, 587-607 (2008)

3. Bortfeld, T., Schlegel, W.: Optimization of beam orientations in radiation therapy: some theoretical considerations. Phys. Med. Biol. 38, 291-304 (1993)

4. Craft, D.: Local beam angle optimization with linear programming and gradient search. Phys. Med. Biol. 52, 127-135 (2007)

5. Custódio, A.L., Rocha, H., Vicente, L.N.: Incorporating minimum Frobenius norm models in direct search. Comput. Optim. Appl. 46, 265-278 (2010)

6. Custódio, A.L., Vicente, L.N.: Using sampling and simplex derivatives in pattern search methods. SIAM J. Optim. 18, 537-555 (2007)

7. Davis, C.: Theory of positive linear dependence. Am. J. Math. 76, 733-746 (1954)

8. Deasy, J.O., Blanco, A.I., Clark, V.H.: CERR: A Computational Environment for Radiotherapy Research. Med. Phys. 30, 979-985 (2003)

9. Dias, J., Rocha, H., Ferreira, B.C., Lopes, M.C.: A genetic algorithm with neural network fitness function evaluation for IMRT beam angle optimization. Cent. Eur. J. Oper. Res. (at press) doi:10.1007/s10100-013-0289-4

10. Lee, E.K., Fox, T., Crocker, I.: Integer programming applied to intensity-modulated radiation therapy treatment planning. Ann. Oper. Res. 119, 165-181 (2003)

11. Li, Y., Yao, D., Yao, J., Chen, W.: A particle swarm optimization algorithm for beam angle selection in intensity modulated radiotherapy planning. Phys. Med. Biol. 50, 3491-3514 (2005)

12. Pugachev, A., Xing, L.: Pseudo beam's-eye-view as applied to beam orientation selection in intensity-modulated radiation therapy. Int. J. Radiat. Oncol. Biol. Phys. 51, 1361-70 (2001)

13. Pugachev, A., Xing, L.: Incorporating prior knowledge into beam orientation optimization in IMRT. Int. J. Radiat. Oncol. Biol. Phys. 54, 1565-74 (2002)

14. Rocha, H., Dias, J.M., Ferreira, B.C., Lopes, M.C.: Beam angle optimization using pattern search methods: initial mesh-size considerations. Proceedings of the 1st International Conference on Operations Research and Enterprise Systems (2012)

15. Rocha, H., Dias, J.M., Ferreira, B.C., Lopes, M.C.: Incorporating Radial Basis Functions in Pattern Search Methods: Application to Beam Angle Optimization in Radiotherapy Treatment Planning. Proceedings of ICCSA 2012, Lecture Notes in Computer Science 7335, 1-16 (2012) 
16. Rocha, H., Dias, J.M., Ferreira, B.C., Lopes, M.C.: Beam angle optimization for intensity-modulated radiation therapy using a guided pattern search method. Phys. Med. Biol. 58, 2939-2953 (2013)

17. Rocha, H., Dias, J.M., Ferreira, B.C., Lopes, M.C.: Selection of intensity modulated radiation therapy treatment beam directions using radial basis functions within a pattern search methods framework. J. Glob. Optim. (at press) doi:10.1007/s10898012-0002-5

18. Romeijn, H.E., Ahuja, R.K., Dempsey, J.F., Kumar, A., Li, J.: A novel linear programming approach to fluence map optimization for intensity modulated radiation therapy treatment planing. Phys. Med. Biol. 48, 3521-3542 (2003)

19. Vaz, A.I.F., Vicente, L.N.: A particle swarm pattern search method for bound constrained global optimization. J. Global Optim. 39, 197-219 (2007) 